\title{
Relationship between tumor-associated macrophage subsets and CD47 expression in squamous cell carcinoma of the head and neck in the tumor microenvironment
}

\author{
Koichi Sakakura ${ }^{1}$, Hideyuki Takahashi ${ }^{1}$, Kyoichi Kaira ${ }^{2}$, Minoru Toyoda', Takaaki Murata ${ }^{1}$, Hiroshi Ohnishi ${ }^{3}$, \\ Tetsunari Oyama ${ }^{4}$ and Kazuaki Chikamatsu ${ }^{1}$
}

Tumor-associated macrophages (TAM) have been classified into an immunostimulatory M1 subset against microbes and malignancies, and an immunoregulatory M2 subset that secretes immunosuppressive cytokines in order to repair tissues damaged by malignancies. The infiltration of $\mathrm{M} 2$ in the tumor microenvironment is known to facilitate immunosuppression and tumor-promoting properties. In the present study, we investigated the phagocytic potential of these macrophage subsets in oral squamous cell carcinoma (OSCC) in relation to the expression of CD47, the 'don't eat me' signal against macrophages. The macrophage subsets M1 (induced by GM-CSF and IFN- $\gamma$ ) and M2 (induced by M-CSF and IL-10) were derived from the CD14 ${ }^{+}$cells of healthy donors. Phagocytosis of the CFSE-labeled CD47 ${ }^{+}$cell line HSC-3 by M1/M2 was assessed using flow cytometry and suppressed by an anti-CD47 neutralizing antibody or CD47 siRNA. Furthermore, $\mathrm{CD}^{+} 8^{+}$and $\mathrm{CD}_{163^{+}}$macrophage subset counts infiltrating tumor tissue and the expression of CD47 on cancer cells were examined immunohistochemically in 74 cases of OSCC, and their relationships with clinicopathological parameters or prognoses were determined. The phagocytic potential of $M 1$ was similar to that of $M 2$ in vitro. Phagocytosis by $M 1$ increased in a CD47-dependent manner by the neutralizing antibody and siRNA, but did not in M2. An immunohistochemical (IHC) analysis revealed that the expression of CD47 did not correlate with macrophage subsets in peritumoral tissue or with any clinicopathological parameters; however, the stronger expression of CD47 by cancer cells and larger number of total macrophages/M2 were independently related to shorter survivals. Our results suggest that the expression of CD47 by cancer cells is related to evasion from phagocytosis, particularly that by M1 in vitro. IHC results indicate that various mechanisms are involved in the engulfing potential of TAM subsets in vivo.

Laboratory Investigation (2016) 96, 994-1003; doi:10.1038/labinvest.2016.70; published online 20 June 2016

Homeostatic immune responses are generally based on the balance between type 1 responses against microbial and cancer cells and type 2 responses associated with allergies. ${ }^{1-3}$ Recent studies demonstrated that this clear biphasic model, such as DC1/DC2, was not always applicable to complex in vivo conditions; however, this model appears to be adaptable to the macrophage subsets, M1 and M2. Tumor-associated macrophages (TAM) have been classified into an M1 subset (or immunostimulatory macrophages), which produces Th1 cytokines such as interferon (IFN) $-\gamma$ and proinflammatory cytokines, and an M2 subset (or immunoregulatory macrophage), which secretes IL-10 and other immunosuppressive cytokines in order to repair tissues damaged by inflammation and malignancies. ${ }^{4,5}$ Previous studies demonstrated that the infiltration of M2 into the tumor microenvironment (TME) promoted topical immunosuppression and the propagation of cancer cells. The roles of M2 in TME have been examined more extensively than those of M1, and revealed correlations with worse clinicopathological parameters and poor prognoses in patients with malignancies. ${ }^{6,7}$

CD47, a ligand of signal-regulatory protein- $\alpha(\operatorname{SIRP} \alpha)$, which is mainly expressed on phagocytic cells, is distributed

\footnotetext{
'Department of Otolaryngology-Head and Neck Surgery, Gunma University Graduate School of Medicine, Gunma, Japan; ${ }^{2}$ Department of Oncology Clinical Development, Gunma University Graduate School of Medicine, Gunma, Japan; ${ }^{3}$ Department of Laboratory Sciences, Gunma University Graduate School of Health Sciences, Gunma, Japan and ${ }^{4}$ Department of Diagnostic Pathology, Gunma University Graduate School of Medicine, Gunma, Japan

Correspondence: Dr K Sakakura, M.D., Ph.D, Department of Otolaryngology-Head and Neck Surgery, Gunma University Graduate School of Medicine, 3-39-15 Showa-machi, Maebashi, Gunma 3718511, Japan.

E-mail: koichisakakura@yahoo.co.jp

Received 25 January 2016; revised 6 April 2016; accepted 18 May 2016
} 
not only to red blood cells and neural cells, but also to cancer cells. CD47 acts as a 'don't eat me' signal to inhibit the phagocytic activity of macrophages; therefore, tumor cells have been suggested to evade the host's immune surveillance by expressing CD47 on their surface. ${ }^{8-11}$ In order to overcome this immunological sanctuary, cancer immunotherapies have been developed that block the expression of CD47 with an anti-CD47 monoclonal antibody (mAb) or SIRP $\alpha$ variant, and their clinical application is now being investigated. ${ }^{12-14}$ Although the expression of CD47 by cancer tissues is a critical parameter for predicting the prognosis of patients with malignancies, most studies have evaluated the mRNA, but not protein of CD47 in patient samples. ${ }^{10,15}$ Although an appropriate $\mathrm{mAb}$ to assess $\mathrm{CD} 47$ protein expression in formalin-fixed paraffin-embedded (FFPE) human samples does not currently exist, several recent studies performed immunohistochemical (IHC) analyses on human tumor samples using polyclonal antibodies against CD47. ${ }^{16,17}$

In addition to the expression of CD47 by cancer cells, the roles of M2 in TME have been comprehensively investigated; however, the relationship between the expression of CD47 in cancer tissues and macrophage subsets in the peritumoral area remains unclear. Zhang et al. ${ }^{18}$ previously reported migratory, but not phagocytic activity in relation to the expression of CD47 and the M1/M2 context with a series of CD47 IHC analyses on patients with malignancies; however, differences in the potential and mechanisms underlying phagocytosis by M1 and M2 in relation to the expression of CD47 or dynamisms among macrophage subsets in TME have not yet been elucidated in detail. In the present study, we investigated the phagocytic potential of M1 (induced by GM-CSF and IFN- $\gamma$ ) and M2 (induced by M-CSF and IL-10) in squamous cell carcinoma of the head and neck (SCCHN) cells lines with the expression of $\mathrm{CD} 47$ in vitro, and examined the in vivo relationship between the expression of $\mathrm{CD} 47$ by oral squamous cell carcinoma (OSCC) and local infiltration of macrophage subsets in relation to clinicopathological parameters.

\section{MATERIALS AND METHODS \\ Cell Culture}

The SCCHN cell line HSC-2, HSC-3, HSC-4, and Ca9-22 were purchased from the Japanese Collection of Research Bioresources (JCRB) Cell Bank (Saito, Japan), and the SCCHN cell line Gun-1 was established and wellcharacterized in our laboratory. ${ }^{19}$ The cells were maintained in RPMI-1640 supplemented with $10 \%(\mathrm{v} / \mathrm{v})$ heat-inactivated fetal bovine serum (FBS), 100 units $/ \mathrm{ml}$ penicillin, $100 \mu \mathrm{g} / \mathrm{ml}$ streptomycin, and $2 \mathrm{mM} \mathrm{L}$-glutamine (all reagents were from Life Technologies, Grand Island, NY, USA). The expression of CD47 on these SCCHN cells were verified with anti-human CD47-PE mAb (Santa Cruz, Dallas, TX, USA) using a flow cytometer as described below. Heparinized venous blood $(20 \mathrm{ml})$ was obtained from healthy subjects with written informed consent, and peripheral blood mononuclear cells (PBMCs) were isolated by centrifugation over Ficoll-Hypaque gradients (Amersham Biosciences, Uppsala, Sweden), washed, and counted in the presence of Trypan blue dye. Monocytes were positively selected using CD14 MicroBeads with an MS column (Miltenyi Biotec, Cologne, Germany), and seeded on a 12-well plate at 0.3 million cells $/ 1 \mathrm{ml} /$ well. The M1 subset was induced from $\mathrm{CD}_{14}{ }^{+}$monocytes by $100 \mathrm{ng} / \mathrm{ml} \mathrm{GM-CSF}$

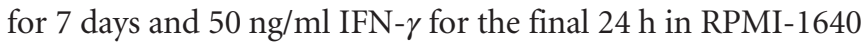
with $10 \%$ FBS, penicillin/streptomycin, and L-glutamine. $\mathrm{CD}_{1} 4^{+}$cells were also incubated with $50 \mathrm{ng} / \mathrm{ml} \mathrm{M}$-CSF for 7 days and $100 \mathrm{ng} / \mathrm{ml} \mathrm{IL-10}$ for the final $24 \mathrm{~h}$ in order to induce the M2 subset (all recombinant human cytokines were from Miltenyi Biotec).

\section{In vitro Phagocytosis Assay}

On day 7 of the macrophage culture, the SCCHN cell line HSC-3 was labeled with $0.5 \mu \mathrm{M}$ CFSE (CellTrace, Life Technologies) according to the manufacturer's instructions. Cells were extensively washed and co-cultured with each macrophage subset for $3 \mathrm{~h}(\mathrm{E}: \mathrm{T}=1: 1)$. After the co-culture, the cells were harvested using a dissociation reagent (TrypLE Express, Life Technologies) and scrapers, followed by blocking of the Fc receptor with Human BD Fc Block (BD Biosciences, San Jose, CA, USA). Surface molecules were stained with mouse anti-human CD14-APC, HLA-DR-PE, CD86-PE, CD163-PE, and CD33-APC mAbs (all antibodies were from BD Biosciences) to macrophage subsets, whereas intracellular staining was conducted with APC-conjugated mouse anti-human CD68 mAb using the BD Cytofix/ Cytoperm Kit (BD Biosciences). A flow cytometric assay was performed using the Attune Acoustic Focusing Cytometer (Life Technologies), and data were analyzed by FlowJo Data Analysis Software (FlowJo, LLC, Ashland, USA). At least 10000 events (actually 12000-14 000 events per flow) were acquired in each flow. $\mathrm{CFSE}^{+} \mathrm{CD}_{3}{ }^{+}$cells were regarded as macrophages engulfing live cancer cells. The percentage of phagocytosis was calculated as macrophages engulfing labeled cancer cells per total number of macrophages.

\section{CD47 Blocking and Silencing}

At the time of the co-culture with the macrophage subsets and $\mathrm{CD}_{4} 7^{+}$SCCHN cell line HSC-3, polyclonal sheep antihuman CD47-blocking IgG (AF4670, R\&D Systems, Minneapolis, USA) was applied to media at concentrations of $0,0.5$, and $2 \mu \mathrm{g} / \mathrm{ml}$. In order to silence the CD47 gene in CD47 ${ }^{+}$HSC-3, CD47 siRNA (Santa Cruz) or MISSION siRNA Universal Negative Control (Sigma Aldrich, St. Louis, USA) was transfected in Opti-MEM Medium (Life Technologies) using Lipofectamine RNAiMAX (Life Technologies) according to manufacturers' instructions. CD47-silenced HSC-3 was co-cultured with the macrophage subsets as described above. The expression of CD47 by the SCCHN cell lines was evaluated by the staining of PE-conjugated mouse 
anti-human CD47 mAb (clone B6H12, Santa Cruz) using flow cytometry as described above.

\section{Immunofluorescence Staining}

Macrophages were also cultured for 7 days with the abovedescribed cytokines in chamber slides (Nunc Lab-Tek II Chamber Slide, Nunc, Roskilde, Denmark). On day 7, CFSElabeled HSC-3 was added (E:T = 1:1) and co-cultured with macrophages for $3 \mathrm{~h}$. After the chamber was removed following the manufacturers' instructions, the slide was fixed with $4 \%$ paraformaldehyde at room temperature (RT) for 15 min. Following the application of $0.2 \%$ Triton X (Sigma Aldrich) for $5 \mathrm{~min}$ and washes, the sample was blocked by $1 \%$ BSA for $30 \mathrm{~min}$. The slide was incubated with APCconjugated anti-CD68 $\mathrm{mAb}(10 \mu \mathrm{l} / 100 \mu \mathrm{l})$ at RT for $1 \mathrm{~h}$, washed, and mounted with Fluoroshield Mounting Medium with DAPI (Abcam, Cambridge, UK). Samples were observed and analyzed using the fluorescent microscope, Zeiss Axioscope, and AxioVision software (Carl Zeiss Microscopy GmbH, Jena, Germany).

\section{May-Grunwald-Giemsa Staining}

Macrophages were also cultured for 7 days with the cytokines in chamber slides as described before. The slides were rapidly dried well in the air, and then fixed by May-Grunwald solution (Muto Pure Chemicals, Tokyo, Japan) for 2 min at RT. Equal volume of phosphate buffer pH 6.4 (Muto Pure Chemicals) was applied on the slides with May-Grunwald solution for $2 \mathrm{~min}$ at RT. After a 20-s rinse with Giemsa solution (Muto Pure Chemicals) diluted by phosphate buffer $\mathrm{pH}$ 6.4, the slides were incubated with Giemsa solution for $15 \mathrm{~min}$ at RT. Following short rinse by distilled water, the samples were rapidly dried well in the air, immersed in xylene, and mounted with the non-aqueous mounting medium DPX (Merck, Darmstadt, Germany).

\section{Patients}

Seventy-four OSCC samples resected surgically at Gunma University Hospital between November 2000 and January 2012 were analyzed. All samples were primary tongue cancer. Patients who received preoperative neoadjuvant chemotherapy or radiation were excluded. All surgical specimens were classified according to the WHO classification by a pathologist who was blind to the clinical findings, and were diagnosed as squamous cell carcinoma. Pathological tumornode-metastasis (TNM) stages were established using the International System for Staging adopted by the American Joint Committee on Cancer and the Union Internationale Centre le Cancer (UICC). Clinicopathological variables, including age, sex, the primary tumor, nodal metastasis, the TNM stage, histological grade, lymphatic/vascular invasion, and p53/Ki-67 staining, as well as overall survival (OS) and progression-free survival (PFS), were evaluated. This study was approved by the Institutional Review Board of Gunma
University (No. 12-12), and was performed in line with the Declaration of Helsinki of 1996.

\section{IHC Staining}

Surgical specimens were fixed in 10\% formaldehyde and routinely processed for paraffin embedding. Serial histological sections $(5-\mu \mathrm{m}$ thick) were deparaffinized in xylene and hydrated in descending dilutions of ethanol. Antigen retrieval was achieved by autoclaving at $121{ }^{\circ} \mathrm{C}$ for $20 \mathrm{~min}$ in citrate buffer ( $\mathrm{pH}$ 6.0) for CD47 and CD163, or by the application of Proteinase K (Dako, Glostrup, Denmark) at RT for 5 min for CD68 staining. Endogenous peroxidase was blocked by 3\% $\mathrm{H}_{2} \mathrm{O}_{2}$, and sections were then covered with $1 \% \mathrm{BSA} / 5 \%$ normal horse serum at RT for $30 \mathrm{~min}$. Slides were incubated at $4{ }^{\circ} \mathrm{C}$ overnight with polyclonal sheep anti-human $\mathrm{CD} 47$ IgG (AF4670, R\&D Systems; $5 \mu \mathrm{g} / \mathrm{ml}$ ), monoclonal mouse anti-human CD163 mAb (clone 10D6; 1:200; Leica Biosystems, Nussloch, Germany), and monoclonal mouse antihuman CD68 mAb (clone PG-M1; ready-to-use; Dako). Labeled Polymer-HRP anti-mouse/rabbit (Dako) was applied to the slides for CD163 and CD68 at RT for 45 min. Slides for CD47 staining were incubated with polyclonal rabbit antisheep IgG (1:500; Abcam) at RT for $1 \mathrm{~h}$, followed by the Vectastain ABC Kit (Vector Laboratories, Burlingame, USA) at RT for $30 \mathrm{~min}$ according to the manufacturer's instructions. The reaction products were detected by $3,3^{\prime}$-diaminobenzidine (Dako), and then counterstained with Mayer's hematoxylin (Wako Pure Chemical Industries, Osaka, Japan). After being dehydrated by ascending dilutions of ethanol, the slides were immersed in xylene, and mounted with DPX.

\section{Evaluation of IHC Samples}

Slides were evaluated by two independent investigators (K.S. and H.T.) in a blinded manner using the light microscope, Zeiss Axioscope. The immunoreactivity of the CD47 protein was scored as $(+),(+/-)$, and $(-)$ when the percentages of stained tumor cells in an entire cancer tissue in each sample slide were $>75 \%, 25-75 \%$, and $<25 \%$, respectively. Red blood cells and nerve cells were regarded as the internal positive control for CD47. More than four areas of a representative field adjacent to cancer cells were counted at $\times 400$ magnification for $\mathrm{CD}^{+} 8^{+}$and $\mathrm{CD}_{163}{ }^{+}$macrophages, and the average was calculated. The positive infiltration of $\mathrm{CD}^{+} 8^{+}$macrophages was defined as more than $200 \mathrm{CD}^{+} 8^{+}$ cells from an average of more than four $\times 400$ high-power fields (HPF), whereas that of $\mathrm{CD}_{163}{ }^{+}$macrophages was defined as $>90$ per HPF, as described below.

On the basis of the previous studies, the expression of $\mathrm{p} 53$ in $>10 \%$ of tumor cells was defined as positive expression. The highly cellular area of the stained sections was evaluated for ki-67. Approximately 1000 nuclei were counted on each slide, and proliferative activity was assessed as the percentage of Ki-67-stained nuclei (Ki-67 labeling index) in the sample. ${ }^{20}$ 

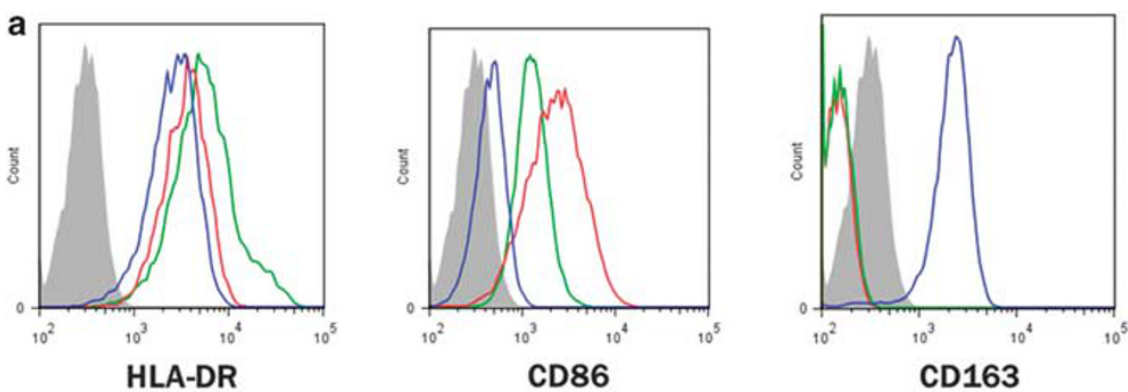

Grey : Isotype control Ab

Green: CD14+monocytes

Red : M1

Blue : M2
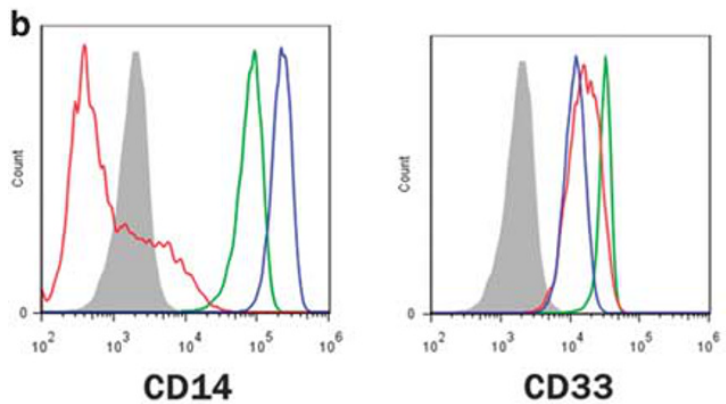

Grey : Isotype control $A b$

Green: CD14+monocytes

Red : M1

Blue : M2

Figure 1 Phenotype analyses of induced M1 and M2 subsets in vitro in Day 7 and positively selected CD14 $4^{+}$monocytes on Day 0 before maturation. (a) $\mathrm{M} 1$ was defined as $\mathrm{CD} 68^{+} \mathrm{HLA}^{-} \mathrm{DR}^{\text {bright }} \mathrm{CD} 86^{+} \mathrm{CD} 163^{-}$, whereas $\mathrm{M} 2$ was HLA-DR ${ }^{\text {dim }} \mathrm{CD} 86^{-} \mathrm{CD} 68^{+} \mathrm{CD} 163^{+}$. These histograms were defined only by scattergram, not by CD68, to compare the expression between macrophages and monocytes. (b) Monocyte marker CD14 and CD33 expression in each subset induced in vitro and in immature $\mathrm{CD} 14^{+}$monocytes. Filled gray means the negative control defined as the CD14- population (sorted using CD14 MicroBeads) stained with the isotype control antibody. Each subset was defined by a scattergram. M2 still expressed CD14, whereas M1 lost the ability to express CD14 after differentiation. CD33 appears to be ideal marker for detecting whole macrophage/monocyte subsets.

\section{Statistical Analysis}

Data were analyzed using the Statistical Package for Social Science version 22.0 (SPSS, IBM, Armonk, USA) and Statcel 3 (OMS Publishing, Tokorozawa, Japan). Mann-Whitney's $U$-test, the Kruskal-Wallis test, the $\chi^{2}$ test for independence, and Fisher's exact test were used to examine differences in continuous and categorical variables. Significance was defined when $P<0.05$. Kaplan-Meier survival curves and log-rank statistics were used to evaluate disease-free survival and OS. Univariate/multivariate regression analyses were performed using the Cox proportional hazards model.

\section{RESULTS}

\section{Phenotypes of Induced Macrophage Subsets}

M1 and M2 subsets were induced for 7 days by GM-CSF ${ }^{+}$ IFN- $\gamma$ and ${\mathrm{M}-\mathrm{CSF}^{+}}^{+} \mathrm{IL}-10$, respectively. Both subsets were stained by the intracellular pan-macrophage marker CD68 as a M1 marker, and the scavenger receptor CD163 as a M2 marker (Figure 1a). M1 was defined as a $\mathrm{CD}^{+} 8^{+} \mathrm{CD} 86^{+}$ $\mathrm{CD}_{163}{ }^{-}$population, whereas $\mathrm{M} 2$ was a $\mathrm{CD}^{+} 8^{+} \mathrm{CD}^{-}{ }^{-}$ $\mathrm{CD}_{163}{ }^{+}$population ${ }^{21-24}$ in this study. M1 expressed the maturation marker and antigen-presenting molecule HLADR more strongly than M2. Therefore, M2 appeared to be a more immature subset than M1. M1 did not express CD14, whereas M2 maintained the expression of CD14, as shown in Figure 1b. CD33 was expressed on each subset and used as a pan-macrophage surface marker in subsequent experiments.

\section{Phagocytosis of CD47 ${ }^{+/-}$SCCHN Cell Lines by M1/M2 Subsets}

The 5 SCCHN cell lines were $\mathrm{CD}_{4} 7^{+}$as confirmed by flow cytometry (Figure 2a). As CD47 expression on HSC-3 was the highest among them, we decided to use this cell line in the following experiments. An immunofluorescent study revealed that CFSE-labeled HSC-3 was engulfed by $\mathrm{CD}^{+} 8^{+}$macrophages induced on a culture slide, as shown in Figure $2 \mathrm{~b}$. 
a
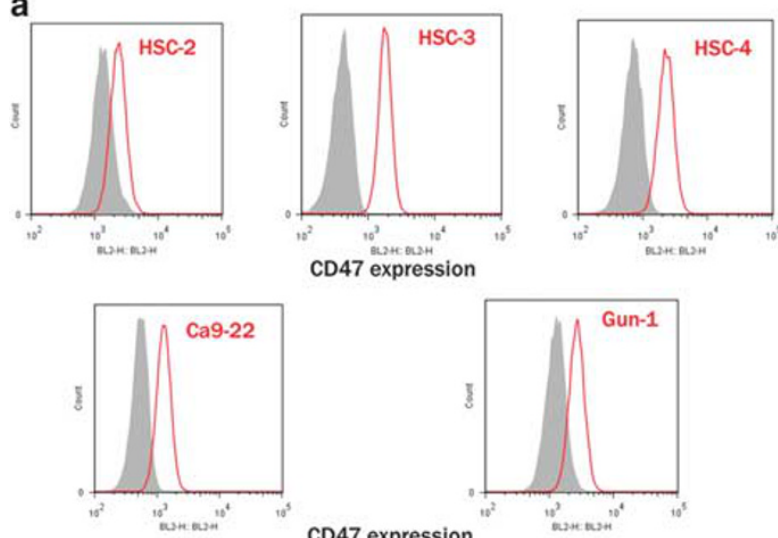

CD47 expression
C
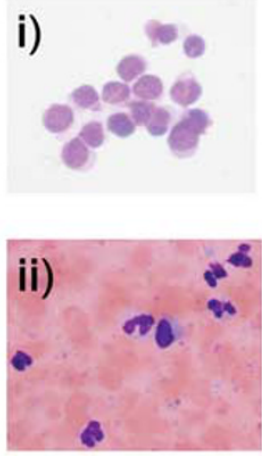

iii)
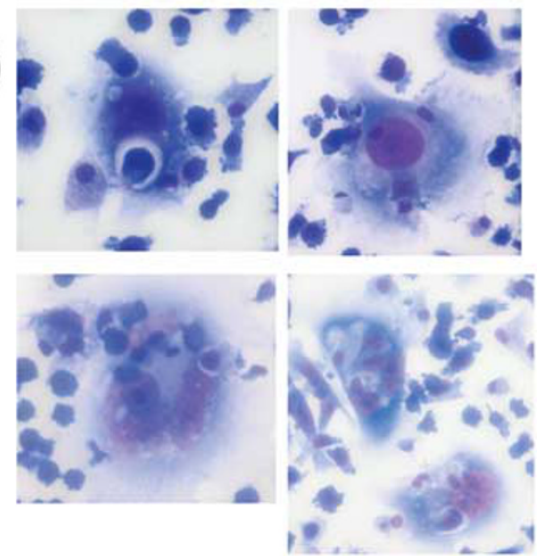

b

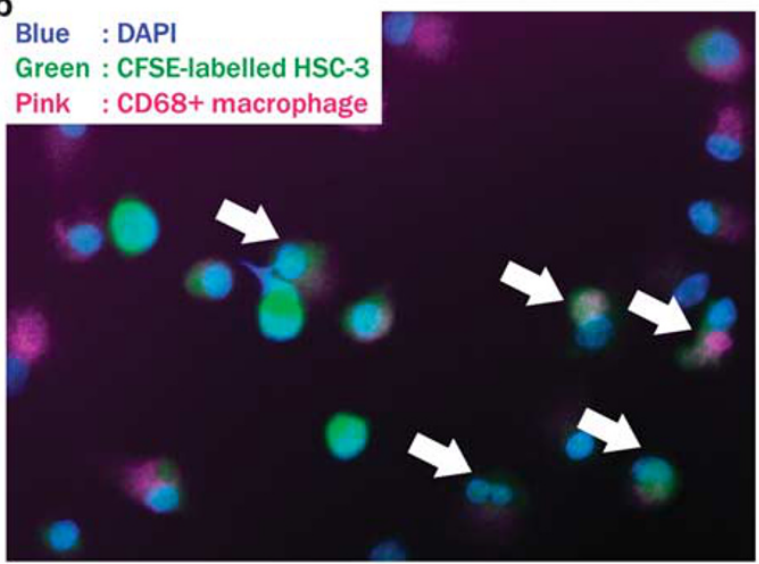

d

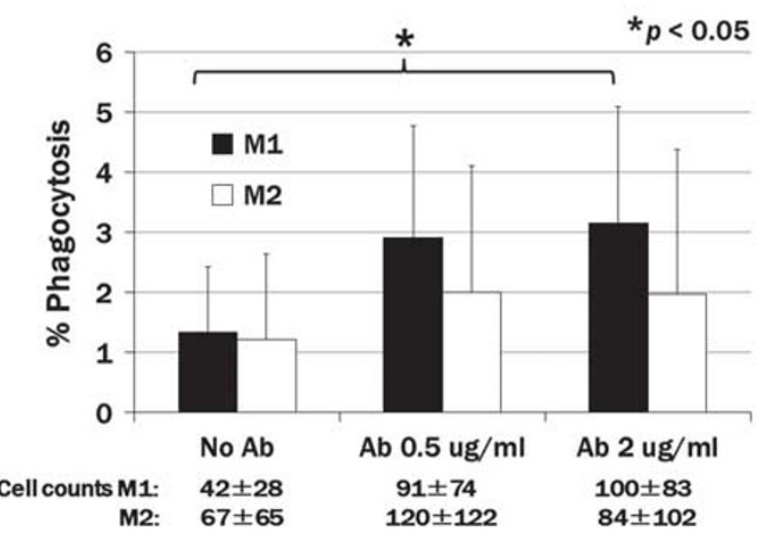

Figure 2 (a) CD47 surface expression in 5 SCCHN cell lines HSC-2, HSC-3, HSC-4, CA9-22, and Gun-1. All 5 cells were expressed CD47, and HSC-3 was the highest. (b) Phagocytosis of CFSE-labeled HSC-3 cells (green) by induced M2 macrophages stained with CD68 (pink) in a culture slide (arrow). Immunofluorescent staining confirmed that cancer cells were engulfed within $3 \mathrm{~h}$ by macrophages induced with our protocol. (c) Phagocytosis of HSC-3 cells by macrophages visualized by May-Grunwald-Giemsa staining. (i) A smear of HSC-3 cell suspension. HSC-3 cells had a small and a relatively reddish nucleus. (ii) A smear of peripheral blood. White blood cells including monocytes had a strong blue nucleus. (iii) Co-culture of induced macrophages and HSC-3 for $4 \mathrm{~h}$. Large macrophages engulfed HSC-3 with small nuclei in vesicles in cytoplasm of the macrophages. (d) Phagocytosis of CD47 ${ }^{+}$HSC-3 by induced M1 (black bar) and M2 (white bar) subsets in vitro. A polyclonal sheep anti-human CD47-blocking lgG was added to media at concentrations of $0,0.5$, and $2 \mu \mathrm{g} / \mathrm{ml}$ at the time of the co-culture with macrophages and the CD47 ${ }^{+} \mathrm{SCCHN}$ cell line HSC-3. Summary experiments were repeated 8 times. Whole actual cell counts for analyses were $8162 \pm 695$ of M1 and $7393 \pm 667$ of M2.

However Jaiswal et al. ${ }^{8}$ quantified the phagocytosis of tumor cells by macrophages in culture flasks with immunofluorescent staining, we could not evaluate a sufficient number of cells using a series of merged fluorescent images as they reported. The phagocytosis of HSC-3 by macrophages in our experimental system was also visualized with May-Grunwald-Giemsa staining in Figure 2c. SCCHN cells with small nucleus were found within vesicles in large macrophages. Figure $2 \mathrm{~d}$ in supplemental material shows the phagocytosis of CD47 ${ }^{+}$HSC-3 by the induced M1 and M2 subsets in vitro. No significant differences were observed in the phagocytic potential of live cancer cells between M1 and M2 despite experiments being repeated 8 times. An anti-human $\mathrm{CD} 47$-blocking $\mathrm{Ab}$ was added to media at concentrations of $0,0.5$, and $2 \mu \mathrm{g} / \mathrm{ml}$ at the time of the co-culture with macrophages and the $\mathrm{CD}_{4} 7^{+} \mathrm{SCCHN}$ cell HSC-3. The addition of the anti-CD47-blocking Ab significantly promoted the phagocytosis of $\mathrm{CD}_{4} 7^{+} \mathrm{HSC}-3$ by M1, but not by M2.

Figure 3 shows the phagocytosis of $\mathrm{CD} 47^{+}$HSC- 3 by each macrophage subset with the knockdown of $\mathrm{CD} 47$ using siRNA. The expression of CD47 on HSC-3 was more effectively, but not completely knocked down by CD47 siRNA than by negative siRNA (Figure 3a). The knockdown of CD47 expression slightly enhanced the engulfment of live HSC-3 by M1 and M2 $(P=0.08$, Figure $3 b)$.

As shown in Figure $2 \mathrm{~d}$ and Figure $3 \mathrm{~b}$, the baseline of phagocytosis by each subset differed between neutralizing $\mathrm{Ab}$ $(\sim 1 \%)$ and siRNA $(5 \%)$, and this may have been due to differences in the culture conditions of the cell line. Although we also assessed a synergic effect of both neutralizing $\mathrm{Ab}$ and siRNA, in order to inhibit CD47 function more effectively, 
a

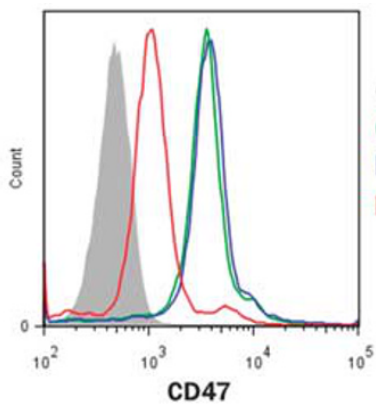

b

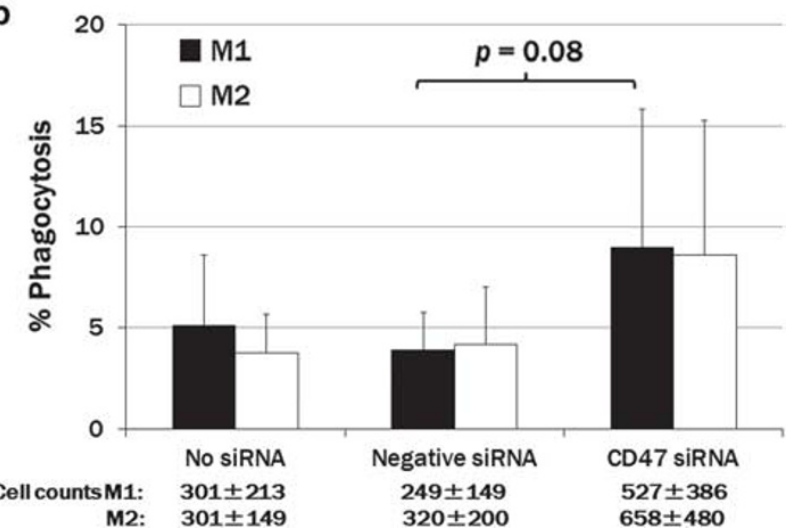

Figure 3 (a) CD47 expression on HSC-3 was more effectively, but not completely, knocked down by CD47 siRNA than by negative siRNA. Gray filled line: stained with isotype control Ab; Green: CD47 staining of HSC-3 without any siRNA; Blue: CD47 expression with negative siRNA; Red: CD47 expression with CD47 siRNA. (b) Slight increases were observed in the phagocytosis of HSC-3 by the induced M1 subset (black bar, $P=0.08$ ), but not by the induced M2 subset (white bar), with the knockdown of CD47 using siRNA in vitro. Summary experiments were repeated in triplicate. Whole actual cell counts for analyses were $8404 \pm 1715$ of M1 and $9683 \pm 839$ of $\mathrm{M} 2$.

the results obtained were very confusing because baselinenegative controls were different between neutralizing $\mathrm{Ab}$ and siRNA due to the difference of culture medium (data not shown).

\section{Patient Characteristics}

The clinicopathological characteristics of all 74 patients are summarized in Table 1. Post-operative adjuvant chemotherapy with the oral administration of S-1 (Taiho Pharmaceutical, Tokyo, Japan), tegafur, or docetaxel was given to 9, 10, and 3 patients, respectively. The median follow-up duration was 915 days (range, 85-3452 days).

\section{Expression of CD47, CD68, and CD163 in Patient Samples}

Figure 4 shows representative IHC staining of CD47 (Figure 4a-c), CD68 (Figure 4d), and CD163 (Figure 4e). As reported previously, ${ }^{11} \mathrm{CD} 47$ was strongly expressed on red blood cells, which were regarded as the internal positive control. The results of IHC staining are summarized in Table 2. CD47 was expressed on approximately half $(56.8 \%)$ of OSCC tissues.
Table 1 Clinicopathological features of patients

\begin{tabular}{|c|c|}
\hline Parameters & $\mathrm{n}=74(\%)$ \\
\hline Age & 33-92, median: 69 years \\
\hline \multicolumn{2}{|l|}{ Sex } \\
\hline Male & $27(36.5)$ \\
\hline Female & $47(63.5)$ \\
\hline \multicolumn{2}{|l|}{ TNM } \\
\hline $\mathrm{T} 1 / \mathrm{T} 2$ & $63(85.1)$ \\
\hline $\mathrm{T} 3 / \mathrm{T} 4$ & $11(14.9)$ \\
\hline No & $53(71.6)$ \\
\hline $\mathrm{N}+$ & $21(28.4)$ \\
\hline Mo & $73(98.6)$ \\
\hline M1 & $1(1.4)$ \\
\hline \multicolumn{2}{|l|}{ Stage } \\
\hline$|/| \mid$ & $47(63.5)$ \\
\hline III/IV & $s 27(36.5)$ \\
\hline \multicolumn{2}{|l|}{ Differentiation } \\
\hline Well/moderate & $66(89.2)$ \\
\hline Poorly & $8(10.8)$ \\
\hline \multicolumn{2}{|l|}{ Invasion } \\
\hline Lymphatic & $35(47.3)$ \\
\hline Vascular & $25(33.8)$ \\
\hline \multicolumn{2}{|l|}{ Adjuvant } \\
\hline Chemotherapy & $22(29.7)$ \\
\hline Radiation \pm chemotherapy & 29 (39.2) \\
\hline Recurrence & $27(36.5)$ \\
\hline Death & $22(29.7)$ \\
\hline
\end{tabular}

TNM, tumor necrosis factor.

The cut-off values of CD68 (200) and CD163 (90) were determined by the statistical largest $P$-values. The expression of CD47 did not correlate $(P>0.05)$ with the infiltration of $\mathrm{CD} 8^{+}$or $\mathrm{CD} 163^{+}$macrophages, lymphatic/vascular invasion, differentiation, lymph node metastasis, clinical stage, T-factor, recurrence, death, vascularization evaluated by CD34 staining, Ki-67, or the p53 status. Although the theoretical qM1 count was calculated by subtracting $\mathrm{CD}_{163}{ }^{+}$cells from $\mathrm{CD}^{+} 8^{+}$cells, the M1 count was also irrelevant to the expression of CD47 by cancer cells and clinicopathological parameters.

\section{Survival Analyses}

Figure 5 shows the relationship between survival and the expression of CD47, CD68, and CD163 by SCCHN tissues from patients. The expression of CD47 by SCCHN tissues correlated with poor OS (Figures 5a, $P=0.015$ ). An increase 

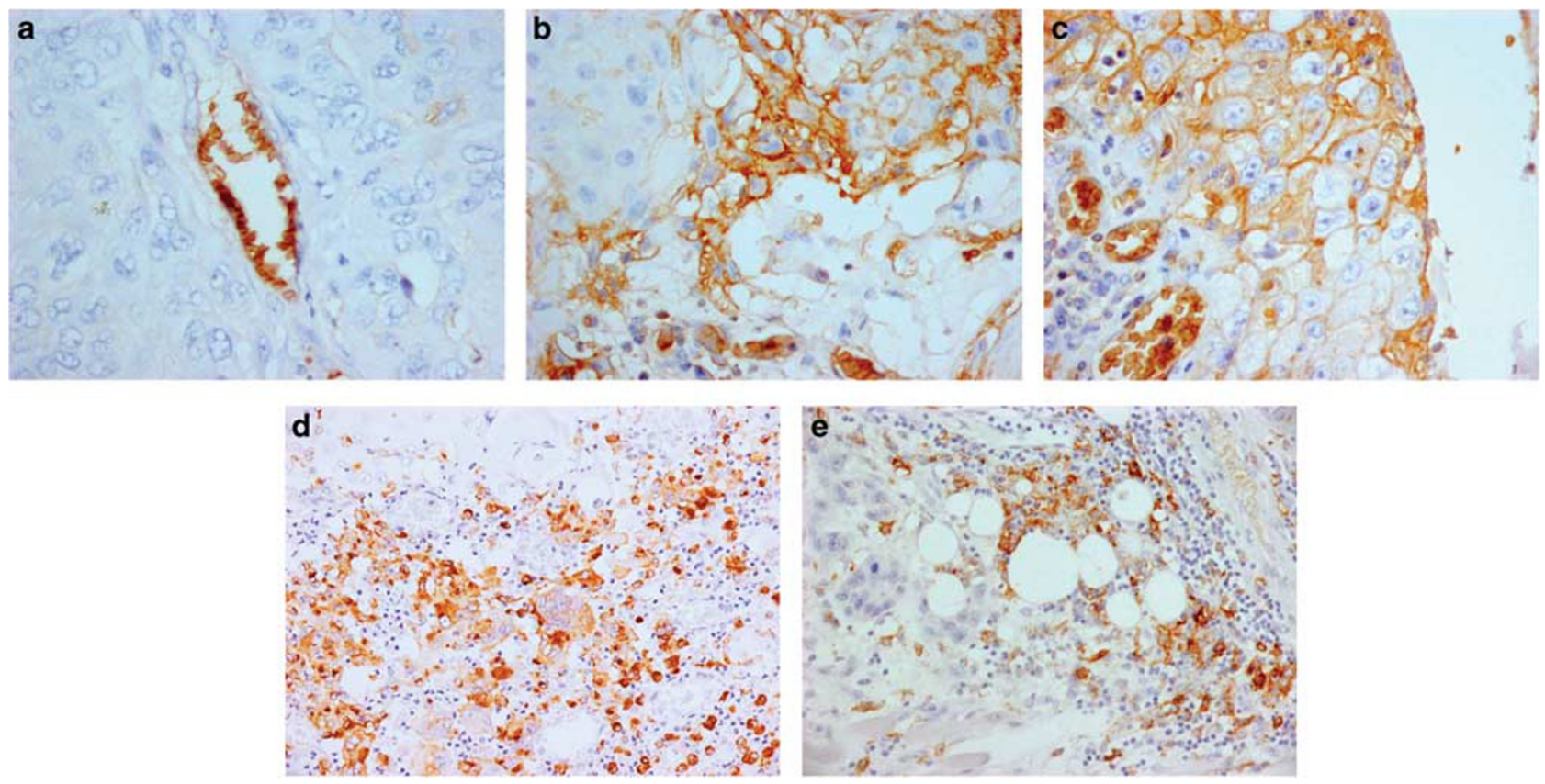

Figure 4 Demonstrative IHC staining of CD47 (a-c), CD68 (d), and CD163 (e) in OSCC tissue magnified by $\times 400$. CD47 staining was evaluated when the percentages of stained tumor cells in an entire lesion were $<25 \%$ as $(-)$. (a), $25-75 \%$ as $( \pm)(\mathbf{b})$, and $>75 \%$ as (+) (c). (a) No stained cancer cells, but positive red blood cells. (b) The upper left tumor population was $\mathrm{CD}^{-} 7^{-}$, whereas the upper right population was $\mathrm{CD}^{2} 7^{+}$in a membranous manner. (c) Most cancer cells were CD47 . More than 4 areas of a representative field adjacent to cancer cells were counted at $\times 400$ magnification for CD68 ${ }^{+}$ (d) and $\mathrm{CD}_{163}{ }^{+}$(e) macrophages and the average was calculated. Each staining of CD44, CD68, and CD163 was from sequential sections.

\section{Table 2 Results of IHC staining}

\begin{tabular}{lcc}
\hline IHC staining & Cut-off value & $\mathrm{n}=74$ (\%) \\
\hline CD47 & $(-)$ & $32(43.2)$ \\
& $( \pm)$ & $23(31.1)$ \\
& $(+)$ & $19(25.7)$ \\
CD68 & $0-199$ & $36(48.6)$ \\
& $200-$ & $38(51.4)$ \\
CD163 & $0-89$ & $57(77.0)$ \\
& $90-$ & $17(23.0)$ \\
\hline
\end{tabular}

IHC, immunohistochemical staining.

in the infiltration of $\mathrm{CD}^{+} 8^{+}$pan-macrophages into SCCHN tissues correlated with shorter OS (Figures 5b, $P=0.035$ ). Consistent with previous findings, ${ }^{7,17}$ OS (Figures $5 \mathrm{c}$, $P=0.025$ ) and PFS (Figures $5 \mathrm{~d}, P=0.011$ ) correlated with the infiltration of $\mathrm{CD}_{163}{ }^{+} \mathrm{M} 2$. A multivariate regression analysis identified the infiltration of $\mathrm{M} 2$ as the only independent prognostic factor for OS and PFS $(P=0.034$, $P=0.023$, respectively).

\section{DISCUSSION}

TAM in TME facilitates the progression and metastasis of cancer cells by various protumoral functions, ${ }^{5,25}$ such as the expression of Tie-2 for angiogenesis, ${ }^{26}$ the expression of osteonectin, ${ }^{27}$ and cathepsin proteases ${ }^{28}$ for tumor invasion, the mobilization of Treg to TME, ${ }^{29,30}$ the secretion of IL-10, TGF- $\beta,{ }^{25}$ and Arginase I, ${ }^{31}$ and the promotion of extravasation, establishment, and growth at metastatic sites. ${ }^{32}$ Recent immunological studies have indicated that TAM dominantly consists of M2, which skews TME to an immunosuppressive or tumor-progressive condition, ${ }^{3,6}$ and also that M2 have a more defective phagocytic potential for tumor cells than M1. However, few studies have identified actual differences in the phagocytic potential of live cancer cells by M1 and M2. The results of the present study revealed not only the dense infiltration of M1 besides M2 in the peritumoral area in patient samples with IHC staining, but also the similar engulfing potential of M2 to that of M1 with an in vitro assay. Accordingly, both subsets may have similar abilities to engulf live cancer cells; M1 may have some pivotal roles in TME, whereas M2 may also contribute to anti-tumor effects through the phagocytosis of cancer cells. However, it is unclear whether our IHC staining and in vitro experiments directly reflect the complex connections among TME in vivo, and these results are consistent with the criticism that the simple biphasic model of the M1/M2 classification is not suitable for discussing TME. ${ }^{4,5}$ Actually, in the murine system the subtypes are not static and macrophages from one subtype can differentiate into the other type. ${ }^{33}$ The previously reported immunosuppressive or tumor-promoting effects of TAM or M2 may depend on other functions apart from the 

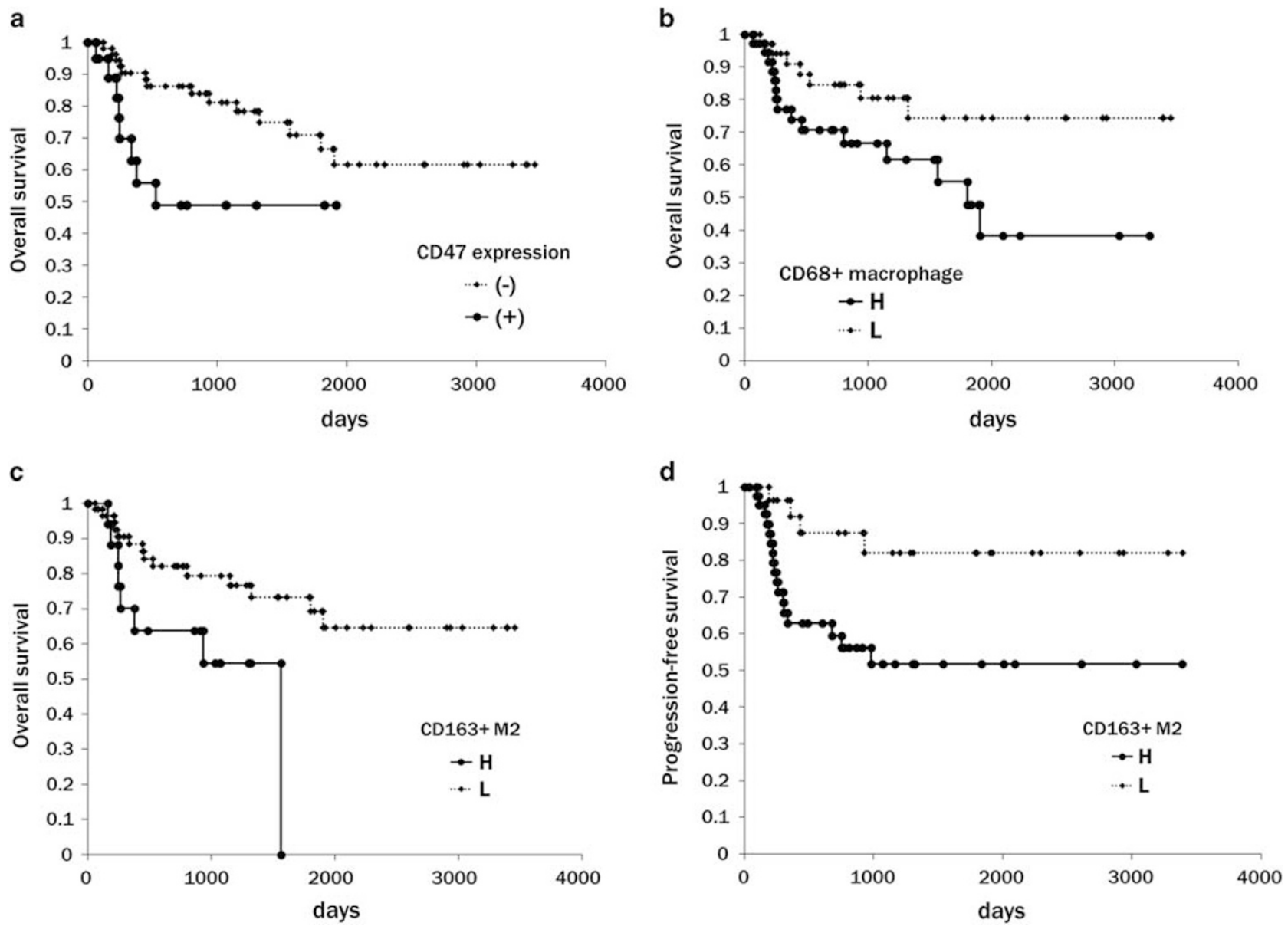

Figure 5 Survival analyses by the Kaplan-Meiermethod. (a) The OS of CD47 $7^{+}$cases ( \pm and +cases) was significantly shorter $(P=0.015)$. (b) Increases in the infiltration of $\mathrm{CD}^{+} 8^{+}$macrophages in SCCHN tissue were associated with shorter OS $(P=0.035)$. (c, d) Overall $(\mathbf{c}, P=0.025)$ and progression-free (d, $P=0.011$ ) survivals correlated with the infiltration of $\mathrm{CD}_{163^{+}} \mathrm{M} 2$.

phagocytosis of live cells, such as cytokine production, invasion, or the engulfment of apoptotic cells.

Although the critical roles of CD47 expressed by cancer cells have been elucidated and applied to therapeutic settings, ${ }^{8-14}$ the actual relationship between $\mathrm{CD} 47^{+}$tumor tissues and TAM subsets in TME in patients remains unclear. Previous studies have been conducted on the functions of CD47 and macrophages using mouse models, and have used CD14 as a macrophage or TAM marker. Although some differences are expected between mice and humans, we showed that CD14 was only expressed by M2, and not by M1 in humans in the present study. Thus, some previous studies may only have recognized the functions of M2 as TAM in TME. ${ }^{12}$ Zhang et al. ${ }^{18}$ investigated differences in the migratory, but not phagocytic activity of $\mathrm{CD} 47^{+}$colon cancer cell lines in the presence of M1 or M2 derived from the human monocytic leukemia cell line THP-1, and showed that M2 migrated to $\mathrm{CD} 47^{+}$cancer cells faster than M1, and also that $\mathrm{CD} 47^{+}$cancer cells invaded faster in the presence of M2 than M1. On the other hand, we attempted to elucidate the phagocytic efficiencies, not invasion or migration, of TAM subsets derived from human PBMCs with the expression of CD47 by live SCCHN cells. Neither M1 nor M2 showed any significant difference in engulfment. Other various mechanisms apart from the surface expression of CD47 on cancer cells may underlie phagocytosis by macrophage subsets; however, engulfment potential among macrophage subsets has not yet been examined. On the other hand, only M1 engulfed the SCCHN cell line in a CD47-dependent manner by the anti-CD47-blocking Ab, as expected. In other words, $\mathrm{CD} 47^{+}$cancer cells mainly inhibited engulfment by M1, but not by $\mathrm{M} 2$, in order to evade phagocytes. In addition, the knockdown of CD47 by siRNA slightly increased phagocytosis by M1 and M2 $(P=0.08)$. One reason why siRNA did not elicit the significant facilitation of phagocytosis may have been due to the incomplete knockdown of CD47 expression, as shown in Figure 3a. Another possibility may be that other molecules were involved in the engulfment of live tumor cells, such as the 'eat me' signal milk fat globule-epidermal growth factor-factor VIII, which also affects phagocytosis by macrophages via integrin $\alpha \mathrm{v} \beta 3 / 5(\mathrm{CD} 51)^{34}$ and induces $\mathrm{M} 2 .^{35}$ 
We also attempted to verify in vitro results by evaluating the IHC expression of CD47 and infiltrating macrophage subset counts in patients' samples. Previous studies demonstrated that CD47 mRNA levels in several malignancies correlated with patient survival. ${ }^{10,15,36}$ However, difficulties have been associated with evaluating CD47 protein expression in tumor FFPE samples from patients, because there is currently no suitable $\mathrm{mAb}$ to stain FFPE samples. Zhang et al. ${ }^{18}$ also performed CD47 IHC staining of FFPE samples using a noncommercial mAb; however, the high concentration of the primary $\mathrm{Ab}$ used, $40 \mu \mathrm{g} / \mathrm{ml}$, may have elicited non-specific background staining, potentially leading to inaccurate findings. Although we also used several commercial mAbs, which were validated for FFPE samples by the manufacturers, none of them successfully stained samples. We finally succeeded in CD47 staining with a sheep anti-human polyclonal $\mathrm{Ab}$ independently, and validated the staining results by strong signals in red blood cells and neural cells as internal controls. Our first IHC results in SCCHN revealed correlations between survival and the expression of CD47 or macrophage subsets independently. Recent studies consecutively reported their IHC findings from FFPE samples collected from breast and ovarian cancer patients also stained with a polyclonal $\mathrm{Ab}$, and found a correlation between the expression of CD47 and patient prognosis, which is consistent with the results of the present study. ${ }^{16,17}$

Our CD47 IHC staining did not reveal any correlation with the infiltration of total macrophages or M2. However, these results were consistent with our in vitro results in which the engulfment of CD47 $7^{+}$SCCHN cells by M2 was not blocked by the anti-CD47-blocking $\mathrm{Ab}$. On the other hand, the mobilization of macrophages, particularly M2, to the cancer bed has been identified as a poor prognostic factor, similar to our study. ${ }^{7,37}$ This may be due not only to the immunosuppressive or tumor-facilitating function of $\mathrm{M} 2$, but also chronic inflammatory TME elicited by cancer cells. ${ }^{38}$ Chronic inflammation by malignancies has been linked to a worse prognosis and has been shown to induce reactive immunosuppressive cells such as M2, cytokines, and surface molecules. ${ }^{39,40}$ Our IHC results of greater macrophage/M2 infiltration to cancer tissue agreed with these widely known phenomena, which were irrelevant to CD47 expression. Nevertheless, our in vitro results suggest the CD47dependent inhibition of M1 phagocytosis. Further studies are needed in order to determine the relationship between CD47 and the macrophage subsets, particularly M1, in vivo.

In conclusion, M1, but not M2 engulfed SCCHN cells in a CD47-dependent manner; however, the phagocytic potential of M2 was similar to that of M1. Further analyses of other surface markers and their functions in TME are currently underway in order to obtain a better understanding of the pivotal roles of macrophage subsets in TME in SCCHN patients.

\section{ACKNOWLEDGMENTS}

This work was supported in part by a Grant-in-Aid for Scientific Research (C) 15K10798 (to KS) and 15K20180 (to TM), Grants-in-Aid for Young Scientists (B) 25861525 (to MT), and a Grant-in-Aid for Challenging Exploratory Research 26670736 (to KC) from the Ministry of Education, Culture, Sports, Science and Technology, Japan. We thank Dr Toshiaki Hikino for his technical assistance with immunohistochemical staining.

\section{DISCLOSURE/CONFLICT OF INTEREST}

The authors declare no conflict of interest.

1. Cherwinski HM, Schumacher JH, Brown KD et al. Two types of mouse helper T cell clone. III. Further differences in lymphokine synthesis between Th1 and Th2 clones revealed by RNA hybridization, functionally monospecific bioassays, and monoclonal antibodies. J Exp Med 1987;166:1229-1244.

2. Fiorentino DF, Bond MW, Mosmann TR. Two types of mouse T helper cell. IV. Th2 clones secrete a factor that inhibits cytokine production by Th1 clones. J Exp Med 1989;170:2081-2095.

3. Mantovani A, Sozzani S, Locati $M$ et al. Macrophage polarization: tumor-associated macrophages as a paradigm for polarized M2 mononuclear phagocytes. Trends Immunol 2002;23:549-555.

4. Qian BZ, Pollard JW. Macrophage diversity enhances tumor progression and metastasis. Cell 2010;141:39-51.

5. Noy R, Pollard JW. Tumor-associated macrophages: from mechanisms to therapy. Immunity 2014;41:49-61.

6. Sica A, Schioppa T, Mantovani A et al. Tumour-associated macrophages are a distinct $\mathrm{M} 2$ polarised population promoting tumour progression: potential targets of anti-cancer therapy. Eur J Cancer 2006;42:717-727.

7. Zhang QW, Liu L, Gong CY et al. Prognostic significance of tumorassociated macrophages in solid tumor: a meta-analysis of the literature. PLoS One 2012;7:e50946.

8. Jaiswal S, Jamieson $\mathrm{CH}$, Pang WW et al. CD47 is upregulated on circulating hematopoietic stem cells and leukemia cells to avoid phagocytosis. Cell 2009;138:271-285.

9. Chan KS, Espinosa I, Chao $M$ et al. Identification, molecular characterization, clinical prognosis, and therapeutic targeting of human bladder tumor-initiating cells. Proc Natl Acad Sci USA 2009;106: 14016-14021.

10. Willingham SB, Volkmer JP, Gentles $A J$ et al. The CD47-signal regulatory protein alpha (SIRPa) interaction is a therapeutic target for human solid tumors. Proc Natl Acad Sci USA 2012;109:6662-6667.

11. Matozaki T, Murata $\mathrm{Y}$, Okazawa $\mathrm{H}$ et al. Functions and molecular mechanisms of the CD47-SIRPalpha signalling pathway. Trends Cell Biol 2009;19:72-80.

12. Majeti R, Chao MP, Alizadeh AA et al. CD47 is an adverse prognostic factor and therapeutic antibody target on human acute myeloid leukemia stem cells. Cell 2009;138:286-299.

13. Chao MP, Weissman IL, Majeti R. The CD47-SIRPa pathway in cancer immune evasion and potential therapeutic implications. Curr Opin Immunol 2012;24:225-232.

14. Weiskopf $\mathrm{K}$, Ring AM, Ho CC et al. Engineered SIRPa variants as immunotherapeutic adjuvants to anticancer antibodies. Science 2013; 341:88-91.

15. Suzuki $\mathrm{S}$, Yokobori $\mathrm{T}$, Tanaka $\mathrm{N}$ et al. CD47 expression regulated by the miR-133a tumor suppressor is a novel prognostic marker in esophageal squamous cell carcinoma. Oncol Rep 2012;28:465-472.

16. Baccelli I, Stenzinger A, Vogel V et al. Co-expression of MET and CD47 is a novel prognosticator for survival of luminal breast cancer patients. Oncotarget 2014;5:8147-8160.

17. Wang $\mathrm{H}$, Tan $M$, Zhang $S$ et al. Expression and significance of CD44, CD47 and c-met in ovarian clear cell carcinoma. Int J Mol Sci 2015;16: 3391-3404.

18. Zhang $\mathrm{Y}$, Sime $\mathrm{W}$, Juhas $\mathrm{M}$ et al. Crosstalk between colon cancer cells and macrophages via inflammatory mediators and CD47 promotes tumour cell migration. Eur J Cancer 2013:49:3320-3334.

19. Chikamatsu K, Sakakura K, Yamamoto T et al. CD4+ T helper responses in squamous cell carcinoma of the head and neck. Oral Oncol 2008;44: 870-877. 
20. Sakakura K, Takahashi H, Kaira $\mathrm{K}$ et al. Immunological significance of the accumulation of autophagy components in oral squamous cell carcinoma. Cancer Sci 2015;106:1-8.

21. Dannenmann SR, Thielicke J, Stöckli M et al. Tumor-associated macrophages subvert T-cell function and correlate with reduced survival in clear cell renal cell carcinoma. Oncoimmunology 2013;2:e23562.

22. Reinartz S, Schumann T, Finkernagel $F$ et al. Mixed-polarization phenotype of ascites-associated macrophages in human ovarian carcinoma: correlation of CD163 expression, cytokine levels and early relapse. Int J Cancer 2014;134:32-42.

23. Yang L, Wang F, Wang L et al. CD163+ tumor-associated macrophage is a prognostic biomarker and is associated with therapeutic effect on malignant pleural effusion of lung cancer patients. Oncotarget 2015;6: 10592-10603.

24. Weber M, Moebius $P$, Büttner-Herold $M$ et al. Macrophage polarisation changes within the time between diagnostic biopsy and tumour resection in oral squamous cell carcinomas-an immunohistochemical study. Br J Cancer 2015;113:510-519.

25. Pollard JW. Tumour-educated macrophages promote tumour progression and metastasis. Nat Rev Cancer 2004;4:71-78.

26. De Palma M, Venneri MA, Galli R et al. Tie2 identifies a hematopoietic lineage of proangiogenic monocytes required for tumor vessel formation and a mesenchymal population of pericyte progenitors. Cancer Cell 2005;8:211-226.

27. Sangaletti S, Di Carlo E, Gariboldi S et al. Macrophage-derived SPARC bridges tumor cell-extracellular matrix interactions toward metastasis. Cancer Res 2008;68:9050-9059.

28. Bonde AK, Tischler V, Kumar $\mathrm{S}$ et al. Intratumoral macrophages contribute to epithelial-mesenchymal transition in solid tumors. BMC Cancer 2012;12:35.

29. Curiel TJ, Coukos G, Zou L et al. Specific recruitment of regulatory $\mathrm{T}$ cells in ovarian carcinoma fosters immune privilege and predicts reduced survival. Nat Med 2004;10:942-949.
30. Ruffell B, Au A, Rugo HS et al. Leukocyte composition of human breast cancer. Proc Natl Acad Sci USA 2011;109:2796-2801.

31. Sharda DR, Yu S, Ray M et al. Regulation of macrophage arginase expression and tumor growth by the Ron receptor tyrosine kinase. J Immunol 2011;187:2181-2192.

32. Qian B, Deng $\mathrm{Y}, \mathrm{Im} \mathrm{JH}$ et al. A distinct macrophage population mediates metastatic breast cancer cell extravasation, establishment and growth. PLoS One 2009;4:e6562.

33. Sica $A$, Mantovani $A$. Macrophage plasticity and polarization: in vivo veritas. J Clin Invest 2012;122:787-795.

34. Hanayama R, Tanaka M, Miwa K et al. Identification of a factor that links apoptotic cells to phagocytes. Nature 2002;417:182-187.

35. Soki FN, Koh AJ, Jones JD et al. Polarization of prostate cancer-associated macrophages is induced by milk fat globule-EGF factor 8 (MFG-E8)-mediated efferocytosis. J Biol Chem 2014;289: 24560-24572.

36. Chao MP, Alizadeh AA, Tang $C$ et al. Anti-CD47 antibody synergizes with rituximab to promote phagocytosis and eradicate non-Hodgkin lymphoma. Cell 2010;142:699-713.

37. Komohara Y, Ohnishi K, Kuratsu J et al. Possible involvement of the M2 anti-inflammatory macrophage phenotype in growth of human gliomas. J Pathol 2008;216:15-24.

38. Asgharzadeh S, Salo JA, Ji L et al. Clinical significance of tumorassociated inflammatory cells in metastatic neuroblastoma. J Clin Oncol 2012;30:3525-3532.

39. Allavena $P$, Mantovani A. Immunology in the clinic review series; focus on cancer: tumour-associated macrophages: undisputed stars of the inflammatory tumour microenvironment. Clin Exp Immunol 2012;167: 195-205.

40. Liu CY, Xu JY, Shi XY et al. M2-polarized tumor-associated macrophages promoted epithelial-mesenchymal transition in pancreatic cancer cells, partially through TLR4/IL-10 signaling pathway. Lab Invest 2013;93:844-854. 